AJMS

28,2

130

Received 5 June 2020

Revised 2 July 2020

7 July 2020

Accepted 9 July 2020

\section{On a class of non-local discrete boundary value problepm}

\author{
Anass Ourraoui \\ Mohammed I University, FSO, Oujda, Morocco, and \\ Abdesslem Ayoujil \\ Center of Trading and Education, Oujda, Morocco
}

\begin{abstract}
Purpose - In this article, the authors discuss the existence and multiplicity of solutions for an anisotropic discrete boundary value problem in $T$-dimensional Hilbert space. The approach is based on variational methods especially on the three critical points theorem established by B. Ricceri.

Design/methodology/approach - The approach is based on variational methods especially on the three critical points theorem established by B. Ricceri.

Findings - The authors study the existence of results for a discrete problem, with two boundary conditions type. Accurately, the authors have proved the existence of at least three solutions.

Originality/value - An other feature is that problem is with non-local term, which makes some difficulties in the proof of our results.
\end{abstract}

Keywords Variational method, Discrete boundary value problem

Paper type Research paper

\section{Introduction}

The non-linear difference equations have been of great interest because of their important applications appearing in various fields of research, such as numerical analysis, non-linear differential equations, computer science, mechanical engineering, control systems, artificial or biological neural networks and social sciences, such as economics. To deal with these kind of problems, a various methods such as fixed points theorems, lower and upper solutions, Browder degree, variational approach and critical point theory have been applied by many different authors. For the recent progress in discrete problems, we refer the readers to valuable monograph by Agarwal [1] and the papers [2,3]. Let $T \geq 2$ be a positive integer, $[a, b]$ be the discrete interval $\{a, a+1, \ldots, b\}$ with $a$ and $b$ are integers such that $a<b$.

In the present paper, we deal with the existence of solutions for the Neumann problem

$$
\left\{\begin{array}{l}
-K(I(u))\left(\Delta\left(\varphi_{p(k-1)}(\Delta u(k-1))\right)+\varphi_{p(k)}(u(k))\right)=\lambda f(k, u(k))+\mu g(k, u(k)), \quad k \in[1, T], \\
\Delta u(0)=\Delta u(T)=0,
\end{array}\right.
$$

as well as for the Dirichlet problem,

\footnotetext{
JEL Classification - 47A75, 35B38, 34L05, 34L30, 35P30

(C) Anass Ourraoui and Abdesslem Ayoujil. Published in Arab Journal of Mathematical Sciences. Published by Emerald Publishing Limited. This article is published under the Creative Commons Attribution (CCBY 4.0) licence. Anyone may reproduce, distribute, translate and create derivative works of this article (for both commercial and non-commercial purposes), subject to full attribution to the original publication and authors. The full terms of this licence may be seen at http://creativecommons. org/licences/by/4.0/legalcode.

The authors would like to thank the anonymous referee for the valuable comments and constructive suggestions.
}

Arab Journal of Mathematical Sciences

Vol. 28 No. 2, 2022

Emerald Publishing Limited e-ISSN: 2588-9214

p-ISSN: 1319-5166

DOI 10.1108/AJMS-06-2020-0003 


$$
\left\{\begin{array}{l}
-K\left(\sum_{k=1}^{T+1}\left(\frac{1}{p(k-1)}|\Delta u(k-1)|^{p(k-1)}\right)\right)\left(\Delta\left(\varphi_{p(k-1)}(\Delta u(k-1))\right)\right)=\lambda f(k, u(k))+\mu g(k, u(k)), \\
\quad k \in[1, T], \\
u(0)=u(T)=0,
\end{array}\right.
$$

Non-local discrete boundary value problem

(2)

where

$$
I(u)=\sum_{k=1}^{T+1}\left(\frac{1}{p(k-1)}|\Delta u(k-1)|^{p(k-1)}\right)+\sum_{k=1}^{T}\left(\frac{1}{p(k)}|u(k)|^{p(k)}\right),
$$

$\Delta u(k)=u(k+1)-u(k)$ is the forward difference operator, $\varphi$ will stand for the homeomorphism defined by $\varphi_{s}(x)=|x|^{s-2} x, K:(0, \infty) \rightarrow(0, \infty)$ be a non-decreasing continuous function, $p:[0, T] \rightarrow[2, \infty)$ is a bounded function while $\lambda, \mu$ are positive real numbers and $f, g$ belong to $\mathfrak{C}$ which is the class of all continuous functions $\mathrm{h}$ which satisfy

$$
|h(k, t)| \leq C_{1}\left(1+|t|^{r(k)-1}\right), \quad \text { for } \operatorname{all}(k, t) \in[1, T] \times \mathbb{R}
$$

with $r:[1, T] \rightarrow[2, \infty)$ being a bounded function.

Equations of this type were suggested by Kirchhoff in 1883. More precisely the following model, which is called Kirchhoff equation, was introduced (see [4])

$$
\rho \frac{\partial^{2} u}{\partial t^{2}}-\left(\frac{P_{0}}{h}+\frac{E}{2 L} \int_{0}^{L}\left|\frac{\partial u}{\partial x}\right|^{2} d x\right) \frac{\partial^{2} u}{\partial x^{2}}=0
$$

where $\rho_{0}, \rho, L$ and h are constants associated to the effects of the changes in the length of strings during the vibrations. It is an extension of the classical D'Alembert's wave equation. A distinguish feature of the above equation is that it contains a non-local coefficient

$$
\frac{P_{0}}{h}+\frac{E}{2 L} \int_{0}^{L}\left|\frac{\partial u}{\partial x}\right|^{2} d x
$$

which depends on the average

$$
\frac{E}{2 L} \int_{0}^{L}\left|\frac{\partial u}{\partial x}\right|^{2} d x
$$

of the kinetic energy $\frac{1}{2}\left|\frac{\partial u}{\partial x}\right|^{2}$ on $[0, \mathrm{~L}]$, and hence the equation is no longer a pointwise identity.

The study of these problems has received more attention. In [2,5-15], a variety of different methods were applied to obtain the existence results to the discrete boundary value problem of the following type

$$
\left\{\begin{array}{l}
-\left(\Delta\left(\varphi_{p}(\Delta u(k-1))\right)\right)=\lambda f(k, u(k)), \quad k \in[1, T], \\
u(0)=u(T)=0,
\end{array}\right.
$$

where $\varphi_{p}(x)=|x|^{p-2}$.

For example, Jiang and Zhou in [16] employing a three critical point theorem, due to Ricceri, established the existence of at least three solutions for perturbed non-linear difference 
AJMS 28,2 equations with discrete boundary conditions. Bonanno and Candito [11], employing critical point theorems in the setting of finite dimensional Banach spaces, investigated the multiplicity of solutions for non-linear difference equations involving the p.Laplacian. Cabada et al. in [2], based on three critical points theorems, investigated different sets of assumptions which guarantee the existence and multiplicity of solutions for difference equations involving the discrete p.Laplacian operator. Candito and Giovannelli [12], using variational methods, established the existence of at least three solutions for the problem above. Far from being exhaustive, further details can be found in [13,17-24].

By taking into account the previous papers and inspired by [25], we study problems (1) and (2) and obtain the existence of three weak solutions by employing a kind of Ricceri's theorem [26]. As for the author's best knowledge, the present papers results are not covered in the related literature, and hence, it is original in its own right.

The structure of this paper is outlined as follows. In Section 2, some preliminary results and statement of main results are presented. In Section 3, the proof of the main results is given.

\section{Preliminaries}

Firstly, we recall some basic properties which will be used in the proof of the precise result.

Through the sequel, we say that the functional $L \in \mathcal{W}_{W}$ if $L: W \rightarrow \mathbb{R}$ possesses the following property: $\left(u_{n}\right)$ is a sequence in $W$ converging weakly to $u \in W$ and $\lim \inf L\left(u_{n}\right) \leq L(u)$, then $u_{n}$ has a subsequence converging strongly to $\mathrm{u}$. When $W$ is finite dimensional, the weak convergence coincides with the strong one.

In order to prove our main results, we will use the following Ricceri's theorem.

Theorem 2.1. [26] Let $W$ be a finite dimensional real Banach space, $\phi \in C^{1}(W, \mathbb{R})$ is coercive and belongs to $\mathcal{W}_{W}$. The derivative of $\phi$ admits a continuous inverse on $W^{*}$; $J: W \rightarrow \mathbb{R}$ a $C^{1}$ functional. Assume that $\phi$ has a strict local minimum $u_{0}$ with $\phi\left(u_{0}\right)=$ $J\left(u_{0}\right)=0$.

Finally, setting assume that

$$
\begin{gathered}
\alpha=\max \left\{0, \limsup _{\|u\| \rightarrow \infty} \frac{J(u)}{\phi(u)}, \limsup _{\|u\| \rightarrow u_{0}} \frac{J(u)}{\phi(u)}\right\}, \\
\beta=\sup _{u \in \phi^{-1}(0, \infty)} \frac{J(u)}{\phi(u)},
\end{gathered}
$$

assume that $\alpha<\beta$.

Then, for each compact interval $[a, b] \subset\left(\frac{1}{\beta}, \frac{1}{\alpha}\right)$ (with the conventions $\frac{1}{0}=\infty, \frac{1}{\infty}=0$ ), there exists $R>0$ with the following property: for every $\lambda \in[a, b]$ and every $C^{1}$ functional $\psi: W \rightarrow \mathbb{R}$ with compact derivative, there exists $\delta>0$ such that, for each $\mu \in[0, \delta]$,

$$
\phi^{\prime}(u)=\lambda J^{\prime}(u)+\mu \psi^{\prime}(u)
$$

has at least three solutions in $W$ whose norms are less than $R$.

Denoting by $F, G:[1, T] \rightarrow \mathbb{R}$ the primitives of $f$ and $g$, i.e.,

$$
F(k, T)=\int_{0}^{T} f(k, s) d s,
$$




$$
G(k, T)=\int_{0}^{T} g(k, s) d s, \quad k \in[0, T], \quad t \in \mathbb{R}
$$

and

$$
\begin{aligned}
p^{+} & :=\max _{k \in[0, T]} p(k), \\
p^{-} & :=\min _{k \in[0, T]} p(k) .
\end{aligned}
$$

Solutions to (1) will be investigated in a space

$$
W=\{u:[0, T+1] \rightarrow \mathbb{R}: \Delta u(0)=\Delta u(T)=0\},
$$

which is a $T$-dimensional Hilbert space, see [5], associated with the norm

$$
\|u\|=\left(\sum_{k=1}^{T+1}|\Delta u(k-1)|^{2}+\sum_{k=1}^{T}|u(k)|^{2}\right)^{\frac{1}{2}} .
$$

It can be verified that for all $u \in W$, one has

$$
\|u\| \geq 1 \Rightarrow \frac{1}{p^{+}}\|u\|^{p^{-}} \leq I(u) \leq \frac{1}{p^{-}}\|u\|^{p^{+}},
$$

and

$$
\|u\| \leq 1 \Rightarrow \frac{1}{p^{+}}\|u\|^{p^{+}} \leq I(u) \leq \frac{1}{p^{-}}\|u\|^{p^{-}} .
$$

We list also some inequalities that will be are used later.

Lemma 2.2. ([8]) For every $u \in W$, we have

1. $\sum_{k=1}^{T}|u(k)|^{m} \leq T(T+1)^{m-1} \sum_{k=1}^{T+1}|\Delta u(k-1)|^{m}, \quad \forall m \geq 2$.

2. $\sum_{k=1}^{T+1}|\Delta u(k-1)|^{p(k-1)} \geq T^{\frac{2-p^{-}}{2}}\|u\|^{p^{-}}-(T+1), \quad$ with $\|u\|>1$.

3. $\sum_{k=1}^{T+1}|\Delta u(k-1)|^{p(k-1)} \geq T^{\frac{p^{+}-2}{2}}\|u\|^{p^{+}}, \quad$ with $\|u\|<1$.

4. $\sum_{k=1}^{T+1}|\Delta u(k-1)|^{p(k-1)} \leq 2^{m} \sum_{k=1}^{T}|u(k)|^{m}, \quad \forall m \geq 2$.

We say that $u \in W$ is a weak solution of problem (1) if

$$
\begin{aligned}
& K(I(u)) \times\left(\sum_{k=1}^{T+1}\left(|\Delta u(k-1)|^{p(k-1)-2} \Delta u(k-1) \Delta v(k-1)\right)+\sum_{k=1}^{T}\left(\frac{1}{p(k)}|u(k)|^{p(k)-2} u(k) v(k)\right)\right) \\
& \quad=\lambda \sum_{k=1}^{T} f(k, u(k)) v(k)+\mu \sum_{k=1}^{T} g(k, u(k)) v(k),
\end{aligned}
$$

for any $v \in W$. 
AJMS

28,2

Define the functionals

$$
\varphi(u)=\widehat{K}\left(\sum_{k=1}^{T+1}\left(\frac{1}{p(k-1)}|\Delta u(k-1)|^{p(k-1)}\right)+\sum_{k=1}^{T}\left(\frac{1}{p(k)}|u(k)|^{p(k)}\right)\right)
$$

and

$$
J(u)=\sum_{k=1}^{t} F(k, u(k)), \psi(u)=\sum_{k=1}^{T} G(k, u(k))
$$

where

$$
\widehat{K}(t)=\int_{0}^{t} K(s) d s
$$

Let $C_{1}$ and $C_{2}$ be positive constants such that

$$
\sum_{k=1}^{T}|u(k)|^{p^{+}} \leq C_{2}\|u\|^{p^{+}} \text {and } \sum_{k=1}^{T}|u(k)|^{p^{-}} \leq C_{1}\|u\|^{p^{-}}, \forall u \in W .
$$

We make the following assumptions.

$$
K_{0}:=\inf _{t \geq 0} K(t)>0 .
$$

Put

$$
A:=\max \left\{0, \limsup _{\eta \rightarrow 0} \frac{\sup _{k \in[1, T]} F(x, \eta)}{|\eta|^{p^{+}}}\right\}
$$

and

$$
B:=\max \left\{0, \limsup _{\eta \rightarrow \infty} \frac{\sup _{k \in[1, T]} F(k, \eta)}{|\eta|^{p^{-}}}\right\} .
$$

$\left(F_{1}\right)$ The following inequality holds

$$
C_{0} K_{0} p^{+} \max \{0, A, B\}<p^{-} \sup _{\eta \neq 0} \frac{\sum_{k=1}^{T} F(k, \eta)}{\sum_{k=1}^{T+1}|\eta|^{p(k-1)}},
$$

with $C_{0}=C_{1}+C_{2}$.

Now, we provide an example of non-linear term which satisfies $\left(F_{1}\right)$.

Example:

Set $f(k, u)=|u|^{\alpha(k)-1}-|u|^{\beta(k)-2}$ for all $(k, u) \in[1, T] \times \mathbb{R}$ where $\alpha$ and $\beta$ are bounded functions such that $1 \leq \alpha(k)<\beta(k), k \in[1, T]$. Hence,

$$
F(k, u)=\left(\frac{|u|^{\alpha(k)}}{\alpha(k)}-\frac{|u|^{\beta(k)}}{\beta(k)}\right) .
$$


There exists $\xi_{u} \in[1, T]$ which depends on $u$ such that

$$
\begin{aligned}
\frac{\sup _{k \in[1, T]} F(k, u)}{|u|^{p^{-}}} & =\left(\frac{|u|^{\alpha\left(\xi_{u}\right)-p^{-}}}{\alpha\left(\xi_{u}\right)}-\frac{|u|^{\beta\left(\xi_{u}\right)-p^{-}}}{\beta\left(\xi_{u}\right)}\right) \\
& \leq\left(\frac{|u|^{\alpha\left(\xi_{u}\right)-p^{-}}}{\alpha^{-}}-\frac{|u|^{\beta\left(\xi_{u}\right)-p^{-}}}{\beta^{+}}\right) \\
& =|u|^{\alpha\left(\xi_{u}\right)-p^{-}}\left(\frac{1}{\alpha^{-}}-\frac{1}{\beta^{+}}|u|^{\beta\left(\xi_{u}\right)-\alpha\left(\xi_{u}\right)}\right) .
\end{aligned}
$$

For $|u|$ large enough, we have,

$$
|u|^{\alpha\left(\xi_{u}\right)-p^{-}}\left(\frac{1}{\alpha^{-}}-\frac{1}{\beta^{+}}|u|^{\beta\left(\xi_{u}\right)-\alpha\left(\xi_{u}\right)}\right) \leq 0
$$

Similarly,

$$
\frac{\sup _{k \in[1, T]} F(k, u)}{|u|^{p^{+}}}=\left(\frac{|u|^{\alpha\left(\xi_{u}\right)-p^{+}}}{\alpha\left(\xi_{u}\right)}-\frac{|u|^{\beta\left(\xi_{u}\right)-p^{+}}}{\beta\left(\xi_{u}\right)}\right) .
$$

Since $\alpha(k)<\beta(k)$ and $p^{+}<\alpha^{-}$then

$$
\limsup _{u \rightarrow 0} \frac{\sup _{k \in[1, T]} F(k, u)}{|u|^{p^{-}}}=0 .
$$

Therefore,

$$
\max \left\{0, \limsup _{|u| \rightarrow 0} \frac{\sup _{k \in[1, T]} F(k, u)}{|u|^{p^{+}}}, \limsup _{|u| \rightarrow \infty} \frac{\sup _{k \in[1, T]} F(k, u)}{|u|^{p^{-}}}\right\}=0 .
$$

Besides, for $u$ small enough we have

$$
\sum_{k=1}^{T} \frac{|u|^{(\alpha-k)}}{\alpha(k)}-\frac{|u|^{\beta(k)}}{\beta(k)}>0 .
$$

which means that $\left(F_{1}\right)$ is verified.

Now, we can state the first main result of this article.

Theorem 2.3. Let $f, g \in \mathfrak{C}$. Under the hypotheses $\left(K_{1}\right)$ and $\left(F_{1}\right)$, if we put

$$
\begin{gathered}
\alpha^{*}=\frac{1}{p^{-}} \inf \left\{\frac{\sum_{k=1}^{T+1}|u(k-1)|^{p(k-1)}}{\sum_{1}^{T} F(k, u(k))} ; \sum_{1}^{T+1} F(k, u(k))>0\right\}, \\
\beta^{*}=\frac{1}{K_{0} p^{+} C_{0} \max \{A, B\}} .
\end{gathered}
$$

then for each compact interval $[a, b] \subset\left(\alpha^{*}, \beta^{*}\right)$ there exists a number $\delta>0$ with the following property: for every $\lambda \in[a, b]$ and every $g \in \mathbb{C}$ there exists $\mu^{*}>0$ such that, for each $\mu \in\left[0, \mu^{*}\right]$, problem (1) has at least three weak solutions whose norms are less than $\delta$. 
AJMS

28,2

136

Now, suppose that we have:

$$
\begin{aligned}
& \left(F_{1}^{\prime}\right) \sup _{u \in W} \sum_{k=1}^{T} F(k, u(k))>0 . \\
& \left(F_{2}^{\prime}\right) \limsup _{|\xi| \rightarrow 0} \frac{\sup _{k \in[1, T]} F(k, \xi)}{|\xi|^{p^{+}}} \leq 0 . \\
& \left(F_{3}^{\prime}\right) \limsup _{|\xi| \rightarrow \infty} \frac{\sup _{k \in[1, T]} F(k, \xi)}{|\xi|^{p^{-}}} \leq 0 .
\end{aligned}
$$

Solutions to (2) will be investigated in a space

$$
W=\{u:[0, T+1] \rightarrow \mathbb{R} \text { s.t } u(0)=u(T+1)=0\},
$$

which is a $T$-dimensional Hilbert space, see [1], with the inner product

$$
(u, v)=\sum_{k=1}^{T+1} \Delta u(k-1) \Delta v(k-1), \quad \text { for all } u, v \in W .
$$

Therefore, the associated norm is defined by

$$
\|u\|=\left(\sum_{k=1}^{T+1}|\Delta u(k-1)|^{2}\right)^{\frac{1}{2}}
$$

Also, it is useful to introduce other norms on $W$, namely

$$
|u|_{m}=\left(\sum_{k=1}^{T}|u(k)|^{m}\right)^{\frac{1}{m}}, \quad \forall u \in W \text { and } m \geq 2 .
$$

It can be verified (see [15]) that

$$
T^{\frac{2-m}{2 m}}|u|_{2} \leq|u|_{m} \leq T^{\frac{1}{m}}|u|_{2}, \quad \forall u \in W \text { and } m \geq 2 .
$$

We report our second main result.

Theorem 2.4. Let $f, g \in \mathfrak{C}$ and $\left(K_{1}\right)$ holds. Under the hypotheses $\left(F_{1}^{\prime}\right)-\left(F_{3}^{\prime}\right)$, if we put

$$
\theta^{*}=\inf \left\{\frac{\sum_{k=1}^{T+1} \frac{1}{p(k-1)}|\Delta u(k-1)|^{p(k-1)}}{\sum_{1}^{T+1} F(k, u(k))} ; \quad \sum_{1}^{T+1} F(k, u(k))>0\right\},
$$

then for each compact interval $\Lambda \subset\left(\theta^{*}, \infty\right)$ there exists a number $\delta>0$ with the following property: for every $\lambda \in \Lambda$ and every $g \in \mathfrak{C}$ there exists $\mu^{*}>0$ such that, for each $\mu \in\left[0, \mu^{*}\right]$, problem (2) has at least three weak solutions whose norms are less than $\delta$. 
Example:

Let consider the above example chosen for the function $f$, then we have

$$
\limsup _{|u| \rightarrow \infty} \frac{\sup _{k \in[1, T]} F(k, u)}{|t|^{p^{-}}} \leq 0,
$$

and

$$
\limsup _{|u| \rightarrow 0} \frac{\sup _{k \in[1, T]} F(k, u)}{|t|^{p^{+}}}=0 .
$$

In addition, for $u$ small enough we have

$$
\sum_{k=1}^{T}\left(\frac{|u|^{(\alpha-k)}}{\alpha(k)}-\frac{|u|^{\beta(k)}}{\beta(k)}\right)>0,
$$

which means that $\left(F_{1}^{\prime}\right)$ is satisfied.

For function $g$ such that $g(k, u)=|u|^{\gamma(k)} u$ if $|u| \leq 1$ and $g(k, u)=|u|^{\delta(k)}$ if $|u| \geq 1$ where $p^{+}<\gamma(k)<\delta(k)$ for all $k \in[1, T]$, and then $f$ and $g$ verify the hypothesis in Theorem 2.4.

\section{Proof}

Proof of Theorem 2.3. It is clear that since the functional $\varphi$ is continuously differentiable on a finite dimensional space its Gâteaux derivative is compact with

$$
\phi^{\prime}(u)=K(I(u))\left(\sum_{k=1}^{T+1}|\Delta u(k-1)|^{(k-1)-2} \Delta u(k-1) \Delta v(k-1)+\sum_{k=1}^{T}\left(|u(k)|^{p(k)-2} u(k) v(k)\right)\right.
$$

for all $u, v \in W$. Since $W$ is a Hilbert space and $K$ is continuous and strictly increasing, it follows that $\phi$ belongs to the class $\mathcal{W}_{W}$.

$$
\text { Let }\|u\|>1 \text {, we have } \varphi(u)=\hat{K}(I(u)) \geq K_{0} \frac{\|u\|^{p^{-}}}{p^{+}},
$$

which means that $\phi$ is coercive. It is evident that $u_{0}=0$ is the only global minimum of $\varphi$ and that $\varphi\left(u_{0}\right)=J\left(u_{0}\right)=0$.

In view of $\left(F_{1}\right)$, there exist $0<\rho<1$ and $R>1$ such that

$$
F(x, \xi) \leq(A+\varepsilon)|\xi|^{p^{+}}
$$

for all $k \in[1, T]$ and $|\xi| \leq \rho$,

$$
F(k, \xi) \leq(B+\varepsilon)|\xi|^{p^{-}}
$$

for all $k \in[1, T]$ and $|\xi| \geq R$.

From the fact that $F$ is bounded on each subset of $[1, T] \times \mathbb{R}$, we may choose $r^{-}>p^{+}$and a suitable constant $c_{1}>0$ such that

$$
F(x, \xi) \leq(A+\varepsilon)|\xi|^{p^{+}}+c_{1}|\xi|^{r^{-}}
$$

for all $(x, \xi) \in[1, T] \times \mathbb{R}$. 
Consequently, for $\|u\|<1$ with $r^{-}>p^{+}$,

$$
\begin{aligned}
J(u) \leq & (A+\varepsilon) \sum_{k=1}^{T}|u(k)|^{p^{+}}+c_{1} \sum_{k=1}^{T+1}|u(k)|^{r^{-}} \\
& \leq C_{2}(A+\varepsilon)\|u\|^{p^{+}}+C_{3}\|u\|^{r^{-}}
\end{aligned}
$$

then, using the inequality (5) and the above estimation, we can write

$$
\limsup _{\|u\| \rightarrow 0} \frac{J(u)}{\varphi(u)} \leq C_{2} \frac{(A+\varepsilon)}{K_{0}} p^{+} .
$$

On the other hand, for each $\|u\|>1$, from (8) it yields

$$
\frac{J(u)}{\varphi(u)} \leq \frac{C_{1}(B+\varepsilon)}{\varphi(u)}\|u\|^{p^{-}} .
$$

Thus we have

$$
\limsup _{\|u\| \rightarrow \infty} \frac{J(u)}{\varphi(u)} \leq p^{+} \frac{C_{1}}{K_{0}}(B+\varepsilon) .
$$

Since $\varepsilon$ is arbitrary, so we obtain

$$
\max \left\{\limsup _{\|u\| \rightarrow \infty} \frac{J(u)}{\varphi(u)}, \limsup _{\|u\| \rightarrow 0} \frac{J(u)}{\varphi(u)}\right\} \leq p^{+} \frac{C_{0}}{K_{0}} \max \{A, B\} .
$$

In addition, it is well known that

$$
p^{-} \sup _{\eta \in \mathbb{R} /\{0\}} \frac{\sum_{k=1}^{T} F(k, \eta)}{\sum_{k=1}^{T+1}|\eta|^{p(k-1)}} \leq \sup _{u \in W /\{0\}} \frac{J(u)}{\varphi(u)} .
$$

From $\left(F_{1}\right)$ and the above inequalities (3.4), (3.4) we infer that

$$
\left\{\limsup _{\|u\| \rightarrow \infty} \frac{J(u)}{\varphi(u)}, \limsup _{\|u\| \rightarrow u_{0}} \frac{J(u)}{\varphi(u)}\right\}<\sup _{u \in \varphi^{-1}(0, \infty)} \frac{J(u)}{\varphi(u)} .
$$

Then the assumptions of Theorem 2.3 are satisfied with $u_{0}=0$ and the conclusion is valid for all $g \in \mathfrak{C}$ and each interval include in $\left(\alpha^{*}, \beta^{*}\right)$

Example

Taking $K(t)=a+b t$ for all $t>0$, with $a, b>0$ and set

$$
f(k, u)=h(k)\left(|u|^{r_{1}(k)-2} u-|u|^{r_{2}(k)-2} u\right)
$$

for all $(k, u) \in[1, T] \times \mathbb{R}$, where $h:[1, T] \rightarrow(0, \infty)$ is an arbitrary function, $p^{+}<r_{1}(k)<$ $r_{2}(k), k \in[1, T]$ where $r_{1}, r_{2}:[1, T] \rightarrow[2, \infty)$ are bounded functions. It is clear that $f \in \mathfrak{C}$. Easily we can prove that

$$
\lim _{t \rightarrow \infty} \frac{F(k, u)}{|u|^{p^{-}}} \leq 0, \quad \lim _{t \rightarrow 0} \frac{F(k, u)}{|u|^{p^{+}}}=0
$$


So $f$ satisfies the assumptions of Theorem 2.3 with

$$
\alpha^{*}=\frac{1}{p^{-}}\left\{\frac{\sum_{k=1}^{T+1}|u(k-1)|^{p(k-1)}}{\sum_{1}^{T} F(k, u(k))} ; \sum_{1}^{T+1} h(k)\left(\frac{|u|^{r_{1}(k)}}{r_{1}(k)}-\frac{|u|^{r_{2}(k)}}{r_{2}(k)}\right)>0\right\}
$$

Non-local discrete boundary value problem

and

$$
\beta^{*}=\infty
$$

Proof of Theorem 2.4. Let start by defining $\phi$ as follows:

$$
\varphi(u)=\hat{K}\left(\sum_{k=1}^{T+1}\left(\frac{1}{p(k-1)}|\Delta u(k-1)|^{p(k-1)}\right)\right)
$$

Let $\|u\|>1$, we have

$$
\varphi(u) \geq K_{0} \frac{\|u\|^{p^{-}}}{p^{+}}
$$

which means that $\phi$ is coercive. It is evident that $u_{0}=0$ is the only global minimum of $\phi$ and that $\phi\left(u_{0}\right)=J\left(u_{0}\right)=0$ In view of $\left(F_{2}^{\prime}\right)$, for $\varepsilon>0$ there exist $0<\rho<1$ such that

$$
F(x, \xi) \leq \varepsilon|\xi|^{p^{+}}
$$

for all $x \in[1, T]$ and $|\xi| \leq \rho$. From $\left(F_{3}^{\prime}\right)$ there exists $R>1$ such that

$$
F(x, \xi) \leq \varepsilon|\xi|^{p^{-}}
$$

for all $x \in[1, T]$ and $|\xi| \geq R$. In view of the fact that $\mathrm{F}$ is bounded on each subset of $[1, T] \times \mathbb{R}$, so we may choose that $r^{-}>p^{+}$and for a suitable constant $c_{1}>0$ such that

$$
F(x, \xi) \leq \varepsilon|\xi|^{p^{+}}+c_{1}|\xi|^{r^{-}}
$$

for all $(x, \xi) \in[1, T] \times \mathbb{R}$.

Consequently, for $\|u\|<1$ with $r^{-}>p^{+}$,

$$
\begin{gathered}
J(u) \leq \varepsilon \sum_{k=1}^{T}|u(k)|^{p^{+}}+c_{1} \sum_{k=1}^{T+1}|u(k)|^{r^{-}} \\
\leq C_{2} \varepsilon\|u\|^{p^{+}}+C_{3}\|u\|^{r^{-}}
\end{gathered}
$$

then, using the inequality $(c)$ in Lemma 2.2 and the above estimation, we can write

$$
\limsup _{\|u\| \rightarrow 0} \frac{J(u)}{\varphi(u)} \leq C_{2} \varepsilon p^{+} .
$$

On the other hand, for each $\|u\|>1$, it is well known that there exists $h \in l^{1}([1, T])$ such that

$$
F(x, t) \leq \varepsilon|t|^{p^{-}}+h(x),
$$

for all $(x, t) \in[1, T] \times \mathbb{R}$. It yields there is $C_{1}>0$ such that 


$$
J(u) \leq C_{1}\left(\varepsilon\|u\|^{p^{-}}+1\right) .
$$

Thus, combining (12) and (13), we get

$$
\limsup _{\|u\| \rightarrow \infty} \frac{J(u)}{\varphi(u)} \leq p^{+} C_{2} \varepsilon .
$$

Since $\varepsilon$ is arbitrary and $\beta>0, \alpha=0$, hence, all the assumptions of Theorem 2.3 are satisfied with $\theta^{*}=\frac{1}{\beta}$ and the proof is complete.

The following corollary is a direct application of Theorem 2.4.

Corollary 3.1. let $f: \mathbb{R} \rightarrow \mathbb{R}$ be a continuous function such that

$$
\sup _{t} F(t)>0
$$

where $F(t)=\int_{0}^{t} f(s) d s$,

$$
\begin{gathered}
\limsup _{|t| \rightarrow \infty} \frac{f(t)}{|t|^{r_{1}(k)}}<\infty, r_{1}^{-}>p^{+}, \\
\limsup _{|t| \rightarrow 0} \frac{F(t)}{|t|^{p^{-}}} \leq 0
\end{gathered}
$$

Set

$$
\eta=\frac{1}{p^{-}} \inf \left\{\frac{\sum_{k=1}^{T}|\Delta u|^{p(k)}}{\sum_{k=1}^{T+1} F(u(k))}, u \in W, \sum_{k=1}^{T+1} F(u(k))>0\right\} .
$$

Then for each compact interval $[c, d] \subset[\eta, \infty)$ there exists a number $\rho>0$ with the following property: for every $\lambda \in[c, d]$ and $g$ in $\mathfrak{C}$ there exists $\sigma>0$ such that for $\mu \in[0, \sigma]$ the problem

$$
\begin{gathered}
-\Delta\left(|\Delta u(k-1)|^{p(k-1)-2} \Delta u(k-1)\right)=\lambda f(u(k))+\mu g(k, u(k)), \quad k \in[1, T] . \\
u(0)=u(T+1)=0,
\end{gathered}
$$

has at least three weak solutions whose norms are less than $\rho$

\section{References}

1. Agarwal RP. Difference equations and inequalities. New York: Marcel Dekker; 2000.

2. Cabada A, Iannizzotto A, Tersian S. Multiple solutions for discrete boundary value problems. J. Math. Anal. Appl. 2009; 356: 418-28.

3. Mihăilescu M, Rădulescu V, Tersian. Eigenvalue problems for anisotropic discrete boundary value problems. J. Difference Equ. Appl. 2009; 15: 557-67.

4. Kirchhoff G. Vorlesungen uber mathematische physik: mechanik. Leipzig: Teubner. 1883.

5. Agarwal RP, Perera K, O'Regan D. Multiple positive solutions of singular and nonsingular discrete problems via variational methods. Nonlinear Anal. 2004; 58: 69-73.

6. Avci M, Pankov A. Nontrivial solutions of discrete nonlinear equations with variable exponent. J. Math. Anal. Appl. 2015; 431: 22-33.

7. Avci M. Existence results for anisotropic discrete boundary value problems. EJDE. 2016; (148): $1-11$. 
8. Ayoujil A. On class of nonhomogenous discrete dirichlet problems, Acta Univ. Apulensis Math. Inform. 2014; 39: 1-15.

9. Ayoujil A. On class of discrete boundary value problem via variational methods. Afrika Mat. 2014: 1349-57.

10. Bisci GM, Repovs̃ DD. Existence of solutions for $p^{-}$Laplacian discrete equations. Appl. Math. Comput. 2014; 242: 454-61.

11. Bonanno G, Candito P. Nonlinear difference equations investigated via critical point methods. Nonlinear Anal. 2009; 70: 3180-86.

12. Candito P, Giovannelli N. Multiple solutions for a discrete boundary value problem involving the $p^{-}$Laplacian. Comput. Math. Appl. 2008; 56: 959-64.

13. Kone B, Ouaro S. Weak solutions for anisotropic discrete boundary value problems, J. Difference Equ. Appl. 2010; 18(February): 1-11.

14. Ourraoui A. Existence of solution to a semilinear discrete problem involving $p^{-}$Laplacian. Acta Univ. Apulensis Math. Inform. 2015; (43): 45-51.

15. Yucedag Z. Existence of solution for anisotrpic discrete boundary value problems of Kirchhoff type. Int. J. Differ. Equ. Appl. 2014; 13(1): 1-15.

16. Jiang L, Zhou Z. Three solutions to Dirichlet boundary value prolems for $p$-Laplacian difference equations. Adv. Difference Equ. 2008. Article ID 345916: 10. doi: 10.1155/2008/345916.

17. Anderson DR, Rachunkova I, Tisdell CC. Solvability of discrete Neumann boundary value problems. J. Math. Anal. Appl. 2007; 331: 736-41.

18. Bereanu C, Jebelean P, Serban C. Periodic and Neumann problems for discrete $p(.)^{-}$laplacian. J. Math. Anal. Appl. 2013; 399(1): 75-87.

19. Bian LH, Sun HR, Zhang QG. Solutions for discrete $p^{-}$Laplacian periodic boundary value problems via critical point theory. J. Difference Equ. Appl. 2012; 18(3): 345-55.

20. Cai X, Yu J. Existence theorems of periodic solutions for second-order nonlinear difference equations. Adv. Difference Equ. 2008. Article ID 247071.

21. Galewski M, Głkçb S. On the discrete boundary value problem for anisotropic equation. J. Math. Anal. Appl. 2012; 386: 956-65.

22. Galewski M, Wieteska R. Existence and multiplicity of positive solutions for discrete anisotropic equations. Turk. J. Math. 2014; 38(2): 297-310. doi: 10.3906/mat-1303-6.

23. Ružička M. Flow of shear dependent electrorheological fluids. CR Math. Acad. Sci. Paris. 1999; 329: 393-98.

24. Yang Y, Zhang J. Existence of solution for some discrete value problems with a parameter. Appl. Math. Comput. 2009; 211: 293-302.

25. Ji C. Remarks on the existence of three solutions for the $p(x)$-Laplacian equations, Nonlinear Analysis. 2011; 74: 2908-15.

26. Ricceri B. A further three critical points theorem. Nonlinear Anal. 2009; 71(9): 4151-157.

\section{Corresponding author}

Anass Ourraoui can be contacted at: anas.our@hotmail.com

For instructions on how to order reprints of this article, please visit our website:

www.emeraldgrouppublishing.com/licensing/reprints.htm

Or contact us for further details: permissions@emeraldinsight.com 